\title{
Application en agro-alimentaire des membranes écrans
}

\author{
par \\ P. SCHAEGIS*
}

\section{QU'EST-CE QUE MICROFILTRER ?}

C'est, sous l'action d'un gradient de pression, séparer deux phases (Solide-liquide / Solide-gaz) par passage au travers d'un milieu poreux, se laissant traverser par l'une des phases (liquide ou gaz) et arrêtant l'autre phase (particules) avec une efficacité variable.

La microfiltration d'une façon générale, fait appel à des milieux micro-poreux dont les diamètres de pores s'étalent entre 10 et 0,02 microns (fig. 1).

Plus précisément en microfiltration sur membrane, on utilise des structures matérielles minces (10 à $200 \mu$ d'épaisseur) et poreuses,

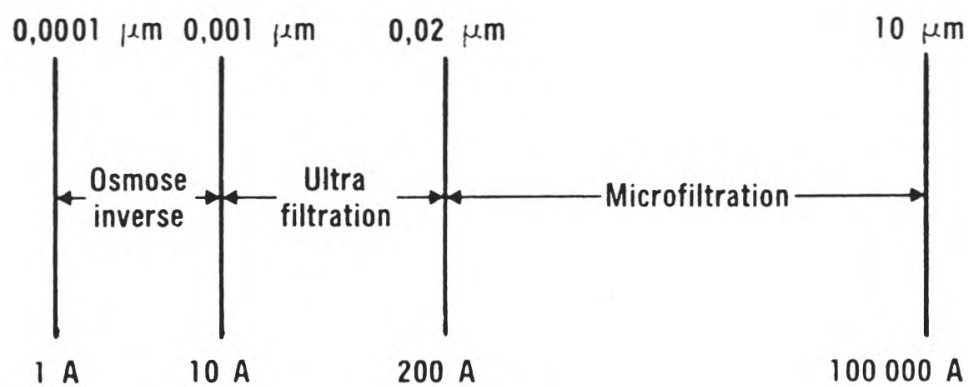

fig. 1

Microfiltration / UF / OI.

Microfiltration - Ultrafiltration - Reverse osmosis.

* D.M.F., 19, rue Marc-Seguin - 75018 Paris. 
dont les diamètres de pores moyens et nominaux les plus couramment utilisés sont $0,1-0,2-0,4-0,6-0,8$ et $1 \mu$.

Les diamètres de pores nominaux sont les diamètres de pores les plus fréquents rencontrés dans le milieu poreux lorsqu'on fait la mesure de la répartition des pores dans la membrane (méthodes de porosimétrie à mercure ou de bulloscopie quantitative).

La mesure de la répartition des pores conduit à tracer la courbe du nombre de pores ayant même diamètre en fonction des diamètres de pores.

On obtient ainsi un spectre de pores plus ou moins étalé ce qui permet de dire que les membranes ont des «bandes passantes » (pour les particules) plus ou moins larges (fig. 2) même si elles ont le même diamètre nominal.

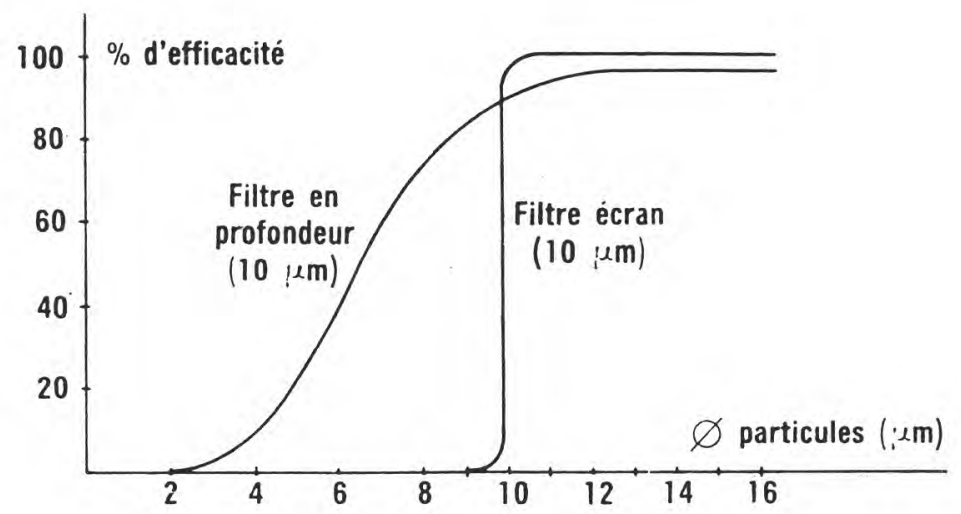

fig. 2

Pouvoir de coupure - Cut-off.

On peut ainsi classer les membranes en fonction du critère de " bande passante » large ou étroite.

\section{MICROFILTRATION A L'AIDE DE MEMBRANES EN SURFACE ET EN PROFONDEUR}

La microfiltration en surface implique l'utilisation de membranes écrans à bande passante étroite qui arrêtent toutes les particules supérieures au diamètre des pores et laissent passer les particules 
inférieures à ce diamètre. Les particules arrêtées sont bloquées sur la surface de la membrane.

La microfiltration en profondeur se caractérise par l'arrêt de la plupart des particules dans la masse de la membrane et plus précisément dans les tortuosités internes.

La bande passsante large dans ce cas signifie que le média capturera des particules de dimensions égales ou supérieures à son diamètre nominal mais capturera aussi grâce à la tortuosité interne, certaines particules inférieures au seuil nominal.

\section{MEMBRANES ECRAN ET MEMBRANES EN PROFONDEUR}

Les membranes en profondeur sont fabriquées principalement à base de nitrate de cellulose ou de mélange d'esters de cellulose bien que d'autres matériaux soient utilisés (tels que nylon, P.T.F.E., etc.) offrant une meilleure résistance chimique.

En général, ces membranes sont réalisées par une méthode consistant à dissoudre le matériau de base dans une solution comportant un ou deux solvants, à étendre la solution sur une bande métallique polie sans fin, et par passage dans différentes étuves, à éliminer les solvants pour créer la porosité.

La membrane ainsi obtenue a une surface relativement rugueuse et on observe facilement au microscope que beaucoup de " passages " sont considérablement plus grands que la dimension nominale des pores annoncée. La bande passante est large.

Ces membranes n'en sont pas moins absolues pendant une période de temps déterminée dépendant du facteur de tortuosité des nombreux canaux de passage.

Quelle que soit la méthode de fabrication de ces membranes et à l'exception des très grosses particules par rapport au seuil, la plupart des particules arrêtées le sont dans la masse poreuse du milieu et non à sa surface.

C'est la raison pour laquelle ces membranes sont dites " en profondeur ".

La membrane écran absolue et submicronique la plus " précise " est une membrane en polycarbonate ou en polyester.

Très mince (10 microns) et très solide, cette membrane possède des pores capillaires cylindriques, presque perpendiculaires par rapport à la surface et dont la précision en diamètre est $+0-10 \%$. La bande passante est donc très serrée.

Cette membrane est fabriquée en deux temps. 
- Le film de polymère est tout d'abord exposé dans un réacteur nucléaire à un bombardement d'ions lourds qui créent dans sa masse une rupture des chaînes moléculaires. La vitesse de défilement du film et l'intensité du rayonnement neutronique définissent le nombre de pores par $\mathrm{cm}^{2}$.

- Les chemins préférentiels ainsi créés par l'irradiation sont agrandis par passage dans divers bains chimiques pour donner naissance à des pores cylindriques parfaitement calibrés. Le temps de passage, en outre, définit le diamètre des pores.

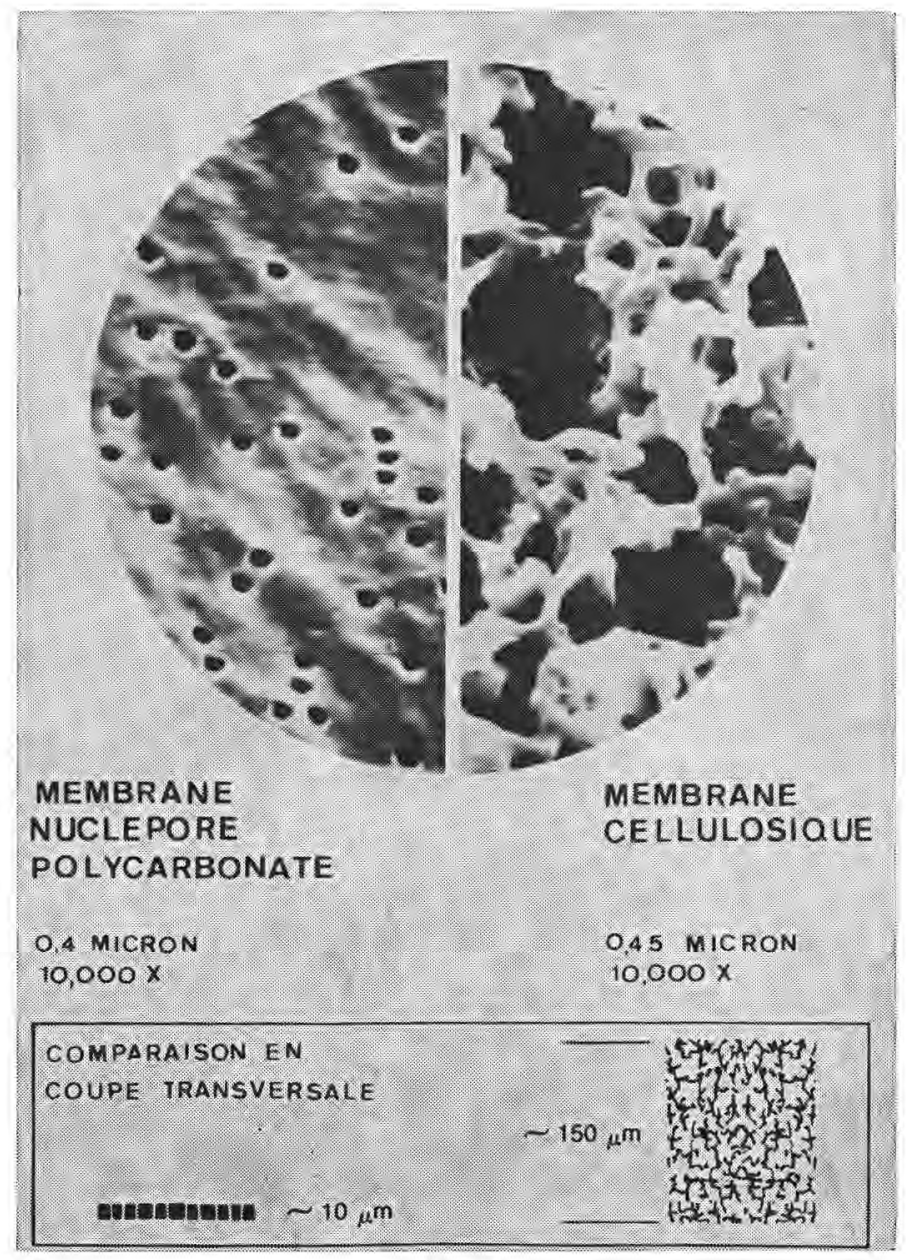

fig. 3

Comparaison entre membrane cellulosique et membrane écran Nuclépore.

Comparison between cellulosic membrane and screen membrane Nuclepore. 


\section{LES CARACTERISTIQUES DES MEMBRANES ECRANS}

Les membranes écrans actuellement sur le marché sont fabriquées soit en polyester, soit en polycarbonate et vendues sous la marque Nuclépore.

Les caractéristiques essentielles de ces membranes sont les suivantes ;

- grande précision de diamètres de pore $\mathrm{d}+0 \%$;

- pores cylindriques et capillaires qui arrêtent les impuretés en surface ;

- grande plage de seuils : de 0,015 à $12 \mu$;

- faible épaisseur de la membrane (10 $\mu)$ permettant de forts débits sous faible perte de charge (une cartouche de $250 \mathrm{~mm}$ de hauteur à $0,2 \mu$ débite $2 \mathrm{~m}^{3} / \mathrm{h}$ d'eau sous une $\Delta \mathrm{P}$ de $200 \mathrm{mb}$ ). Porosité de $20 \%$. Nombre de pores au $\mathrm{cm}^{2}$ : de $10^{6}$ à $10^{9}$;

- grande facilité de plissage, le film n'étant pas fragile, qui permet l'obtention de surfaces filtrantes élevées $\left(3,7 \mathrm{~m}^{2}\right.$ pour une cartouche de $500 \mathrm{~mm}$ de longueur);

- surface lisse permettant un flux tangentiel aisé ;

- symétrie de la membrane ;

- très faibles extractibles permettant un lavage instantané de la membrane ;

- nettoyage très facile à contre courant. Les impuretés supérieures à un seuil donné restant en surface, un contre courant permet de restituer la quasi-totalité de la perméabilité initiale ;

- grande transparence de la membrane qui ne se colore pas et permet ainsi un examen facile au microscope ;

- grande inertie chimique ;

- résistance de la température de $-170^{\circ} \mathrm{C}$ à $+140^{\circ} \mathrm{C}$. Stérilisation à la vapeur facile ;

- membrane hydrophile et non hygroscopique ;

- absorption et adsorption très réduites.

Ces caractéristiques intéressantes sont mises à profit dans l'ensemble des applications industrielles ou de laboratoire que nous allons décrire à présent dans le domaine agro-alimentaire.

En particulier, la bande de répartition des pores de ces membranes assure un arrêt bactérien très efficace qui se maintient dans le temps, contrairement aux filtres à action en profondeur, dans lesquels les bactéries arrivent à passer après un temps plus ou moins long. 


\section{APPLICATIONS DES MEMBRANES A PORES CAPILLAIRES}

\section{a) Applications industrielles}

- Filtration de l'eau à 0,2 4 en continu 24 h sur 24

L'eau pour usage industriel en agro-alimentaire peut être filtrée à grands débits à $0,2 \mu$ absolu.

Ainsi le schéma de la figure 4 montre une installation d'un débit de $60 \mathrm{~m}^{3} / \mathrm{h}$, comportant 3 filtres équipés chacun de 12 cartouches 30 pouces à $0,2 \mu$.

\section{Entrée}

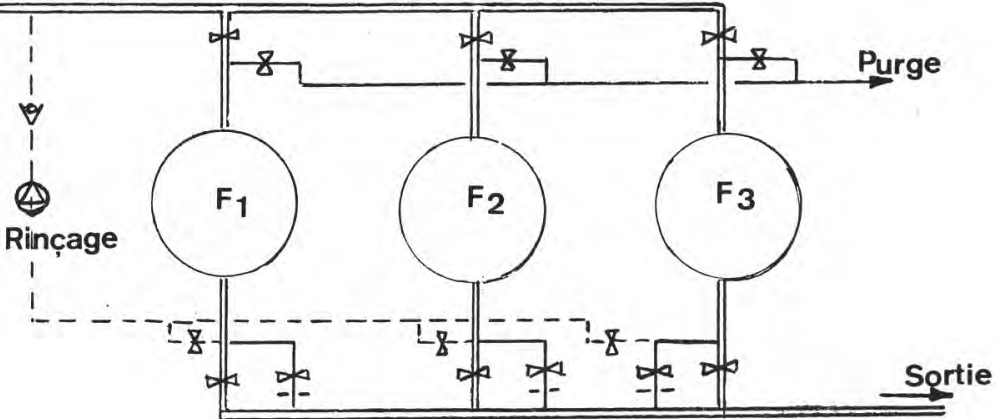

fig. 4

Filtration à décolmatage automatique $\left(60 \mathrm{~m}^{3} / \mathrm{h}\right.$ à $\left.0,2 \mu\right)$ en continu.

Filtration $\left(60 \mathrm{~m}^{3} / \mathrm{h}\right.$ to $\left.0,2 \mu\right)$ with automatic and continuous defouling.

Deux sont en service $\left(30 \mathrm{~m}^{3} / \mathrm{h}\right.$ chacun).

Par exemple F1 et F2. F3 est en arrêt mais propre et plein d'eau. Lorsque la $\Delta \mathrm{P}$ a atteint une valeur fixée sur $\mathrm{F} 1$ et $\mathrm{F} 2$, on ouvre $\mathrm{F} 3$ qui débite et on décolmate d'abord F1 à contre-courant, F2 continuant à débiter avec F3 pour assurer le débit de $60 \mathrm{~m}^{3} / \mathrm{h}$.

L'eau de décolmatage à contre-courant traverse un diaphragme pour limiter la pression en ligne.

Puis on remet F1 en service avec F3, tandis qu'on décolmate F2. Ce dernier reste en attente jusqu'à ce que la $\Delta \mathrm{P}$ sur F1 et F3 aît atteint la valeur fixée pour le décolmatage.

L'opération expliquée ci-dessus se reproduit par permutation circulaire et alors, après décolmatage, ce seront les filtres F2 et F3 qui fonctionneront et ainsi de suite. 
L'ensemble programmé sur automate fonctionne avec des vannes pneumatiques $24 \mathrm{~h}$ sur 24 et distribue une eau filtrée à $0,2 \mu$, à débit constant. La durée d'un décolmatage est de 10 min maximum.

Il est bien entendu que la qualité de l'eau en amont du système doit être bonne et, qu'en particulier, une préfiltration appropriée mais facile doit être faite à 3 ou $5 \mu$.

Le même montage se prête à des filtrations à $0,4-0,6$ ou $0,8 \mu$.

- Filtration de l'eau 0,2 u avec décolmatage à contre-courant en discontinu

Un montage plus simple que celui proposé ci-dessus (voir fig. 5) permet d'assurer un décolmatage à contre-courant en stoppant le débit principal.

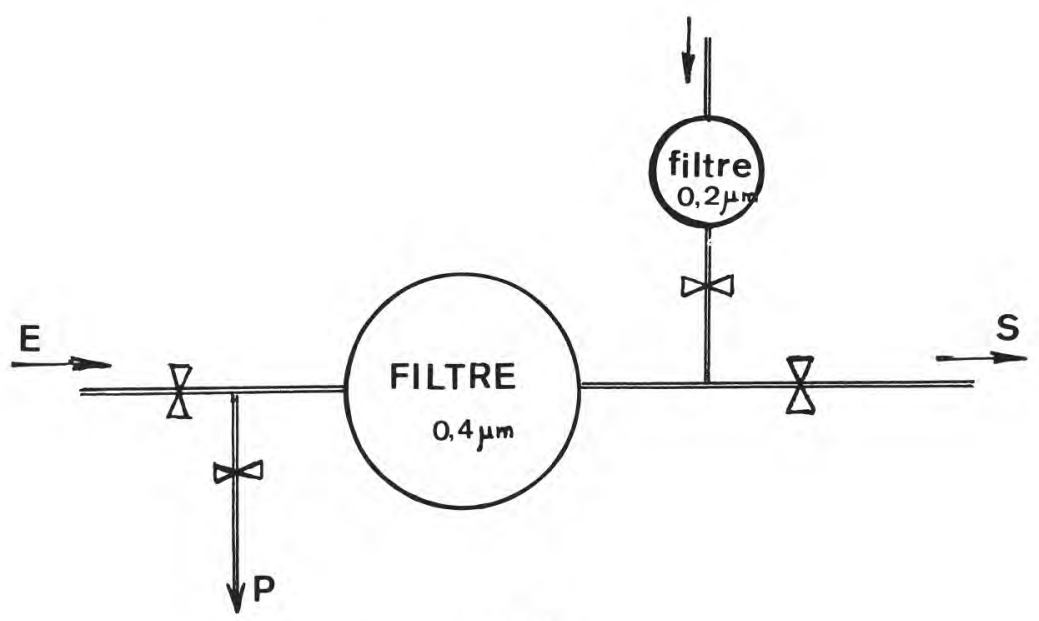

fig. 5

Décolmatage à contre-courant en discontinu.

Discontinuous back-flush defouling.

Le filtre à $0,2 \mu$ ou $0,4 \mu$ débite en ligne par exemple 10 ou $15 \mathrm{~m}^{3} / \mathrm{h}$ (voir fig. 6) et le petit filtre adjoint permet le décolmatage à contre courant par le jeu des vannes et le renvoi à l'égout des impuretés. Mais alors, le débit principal est arrêté pendant $10 \mathrm{~min}$ au plus.

De telles installations en continu ou discontinu permettent d'obtenir une eau pure et de bonne qualité sans pour autant consommer des cartouches filtrantes. Donc l'intérêt économique du procédé est évident. Il est utilisé en filtration d'eau de table ou de préparation de boisson. 


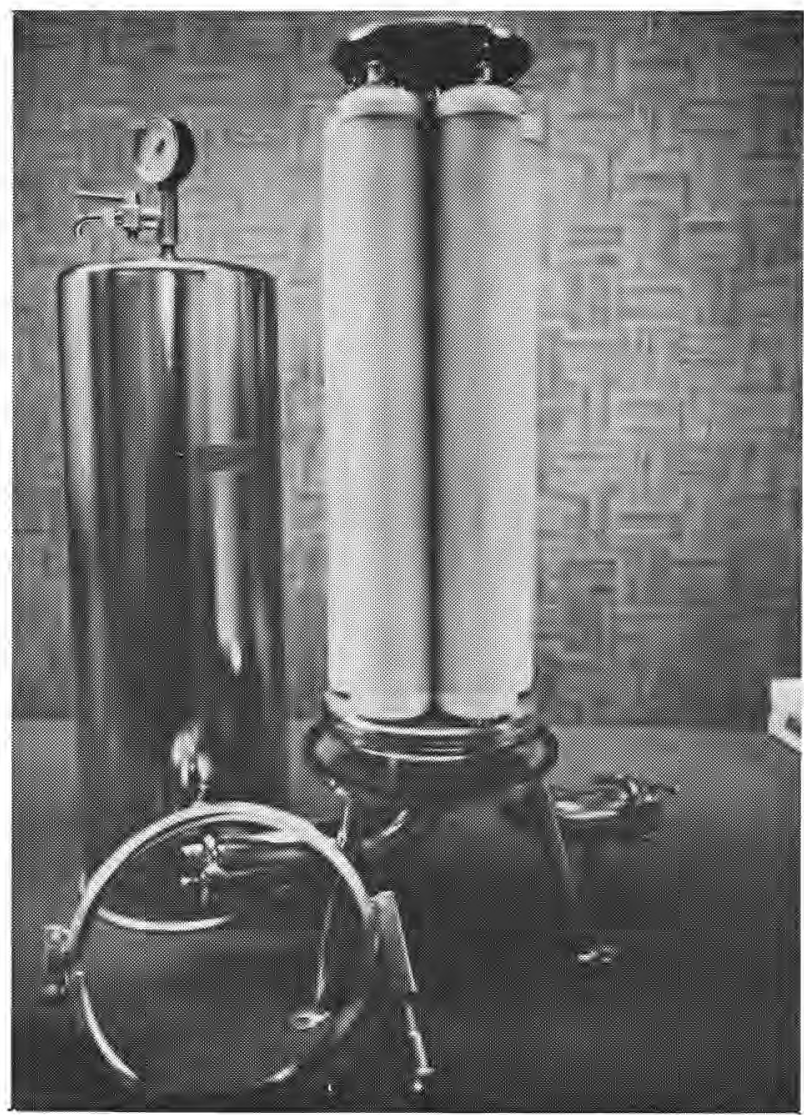

fig. 6

Filtration à $15 \mathrm{~m}^{3} / \mathrm{h}$ avec décolmatage à contre-courant en discontinu. $15 \mathrm{~m}^{3} / \mathrm{h}$ filtration unit with discontinuous back-flush defouling.

- Filtration avant ultrafiltration

Les mêmes dispositifs peuvent être utilisés avant UF, pour des lactosérums par exemple. Les seuils peuvent être 0,4 ou $0,2 \mu$.

Alors le rendement d'UF est considérablement augmenté (20 à $30 \%$ ) et la fréquence des interventions diminuée.

- Filtration des vins et alcools

Les mêmes principes de décolmatage à contre-courant ont été mis en avant pour la filtration des vins et alcools.

Le schéma de la figure 7 explique facilement le principe retenu avec un préfiltre et un filtre final ainsi qu'avec le dispositif de lavage à contre-courant. 
Les seuils de filtration utilisés sont les suivants :

\begin{tabular}{c|c|c}
\hline Type de vin & Préfiltration & Filtration finale \\
\hline Vin rouge & $1 \mu$ & $0,6 \mu$ \\
Vin blanc & $0,8 \mu$ & $0,4 \mu$ \\
\hline
\end{tabular}

GROUPE FILTRATION VIN - 10 à $100 \mathrm{hl} / \mathrm{h}$

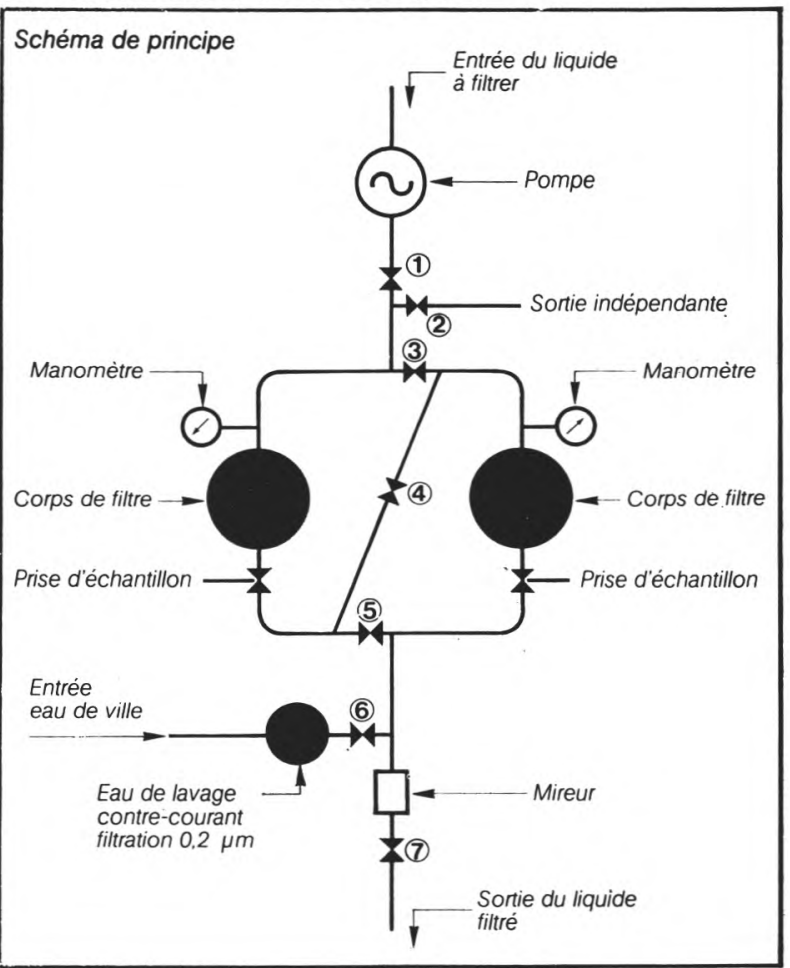

Fonctionnement du groupe

\begin{tabular}{|c|c|c|}
\hline Description & Vannes ouvertes & Vannes fermées \\
\hline $\begin{array}{c}\text { Préfiltration et filtration finale } \\
\text { des vins }\end{array}$ & $1-4-7$ & $2-3-5-6$ \\
\hline Filtration en parallèle & $1-3-5-7$ & $2-4-6$ \\
\hline Nettoyage à contre-courant & $2-3-5-6$ & $\begin{array}{c}1-4-7 . \\
\text { puis ouverture de 4 }\end{array}$ \\
\hline
\end{tabular}

Les caractenstiques e: performarces sont données icia a titre indicatt. SMF Deut étre amené à les modifier sans ans prealable.

fig. 7

Schéma de fonctionnement d'un groupe destiné à la filtration du vin.

Processing line of a wine filtration unit. 
Le vin doit être préalablement correctement filtré sur Kieselghur blanc puis rose de préférence.

L'utilisation préalable de plaques filtrantes est inutile grâce au décolmatage à contre-courant possible du préfiltre.

L'originalité du procédé consiste en effet en un décolmatage aisé à contre-courant et uniquement à l'eau froide des filtres, eau qui a traversé au préalable un petit filtre absolu à $0,2 \mu$.

Les débits sont élevés. Ainsi un groupe à 3 cartouches de $500 \mathrm{~mm}$ débite entre 30 et $40 \mathrm{hl} / \mathrm{h}$. La $\Delta \mathrm{P}$ de départ est de l'ordre de $200 \mathrm{mb}$ et on décolmate en quelques minutes lorsque celle-ci atteint entre 1,5 et 2 bars, selon les types de vin.

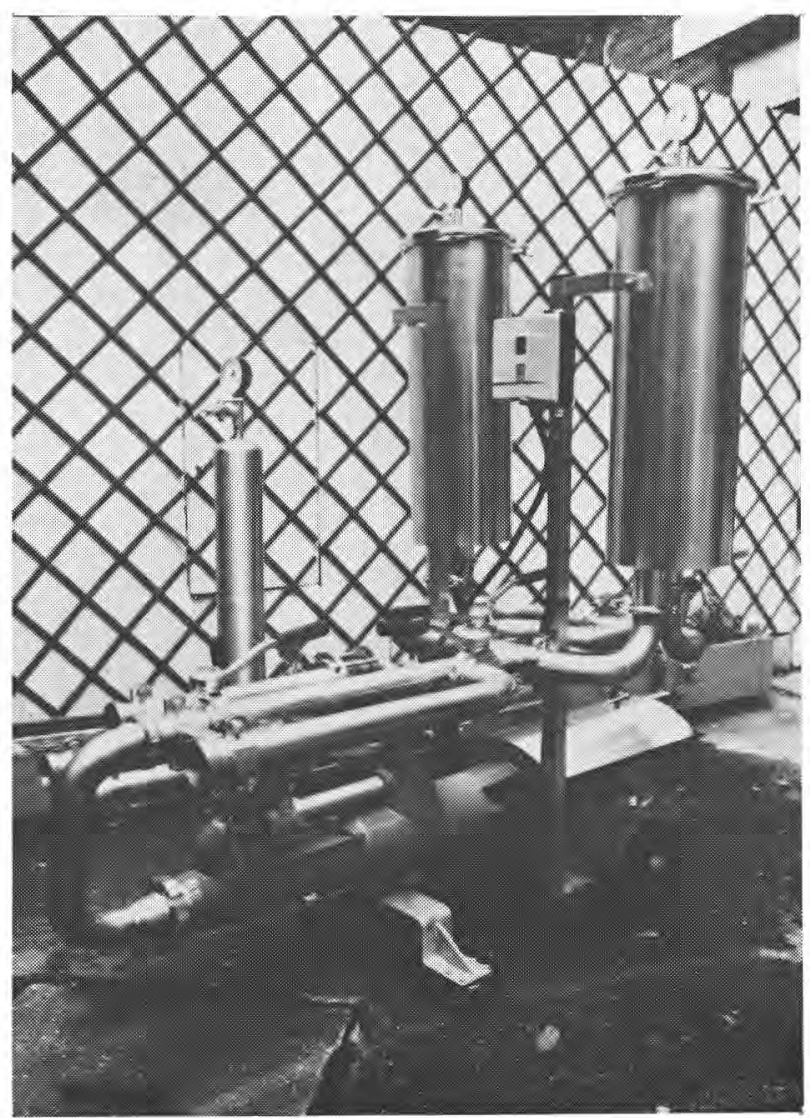

fig. 8

Photo d'un groupe destiné à la filtration du vin.

Wine filtration unit. 
La courbe du volume total filtré en fonction du temps prouve l'intérêt de cette technique très simple et peu onéreuse, puisque son coût, investissement compris, est bien inférieur à $0,7 \mathrm{~F}$ par hectolitre. Une cartouche de $500 \mathrm{~mm}$ peut filtrer plus de $3000 \mathrm{hl}$ avant d'être colmatée. Ces cartouches, moyennant quelques précautions très simples, sont conservables d'une campagne à l'autre.

De plus de tels groupes se présentent sous une forme compacte (fig. 8) où la pompe volumétrique supporte les filtres, les mireurs, les prises d'échantillons stériles et le dispositif de lavage à contrecourant.

Très mobiles, de telles unités de filtration permettent de filtrer de 10 à $210 \mathrm{hl} / \mathrm{h}$, s'adaptent à tous les chais ou installations de mise bord camion, sans altérer le goût des vins.

Pour les alcools, selon leur nature, le seuil final peut être inférieur à celui retenu pour les vins.

Ainsi, un cognac peut être filtré à $0,2 \mu$ sans perdre aucune de ses qualités organoleptiques.

Aucun problème pour la stérilisation à l'eau chaude ou à la vapeur, mais ne jamais oublier que celles-ci doivent être filtrées au préalable à $0,2 \mu$ de préférence. Elle ne doit être effectuée qu'après décolmatage préalable à contre-courant et à froid des cartouches.

Nous avons également sur cartouches à pores capillaires et calibrés, filtré des vins et alcools en filtration tangentielle (voir paragraphe suivant expliquant la technique).

C'est dans la boucle que les impuretés s'accumulent et on peut facilement les concentrer dans une cuve intermédiaire régulièrement purgée. Il faut aussi, à cause de la couche de polarisation, opérer à intervalles donnés un décolmatage des membranes en fermant et ouvrant rapidement les vannes réglant les débits amont et aval de la membrane.

Toutefois, une telle technique présente l'inconvénient majeur de faire circuler le vin, le chauffer et l'aérer.

Pour des débits élevés, l'installation est plus complexe que pour la filtration directe et par suite, plus onéreuse pour de petites ou moyennes exploitations.

- Filtration par la technique dite du flux tangentiel

Le schéma de la figure 9 ci-après indique le principe de cette technique que nous pratiquons sur cartouche plissée spéciale.

Cette technique consiste à entourer la cartouche d'une gaine qui laisse les plis, dans leur partie supérieure et inférieure, accessibles au fluide.

Cette cartouche, bouchée à sa partie basse, est placée dans un corps de filtre et un joint étanche médian empêche le liquide brut de passer directement du haut en bas du corps de filtre. 


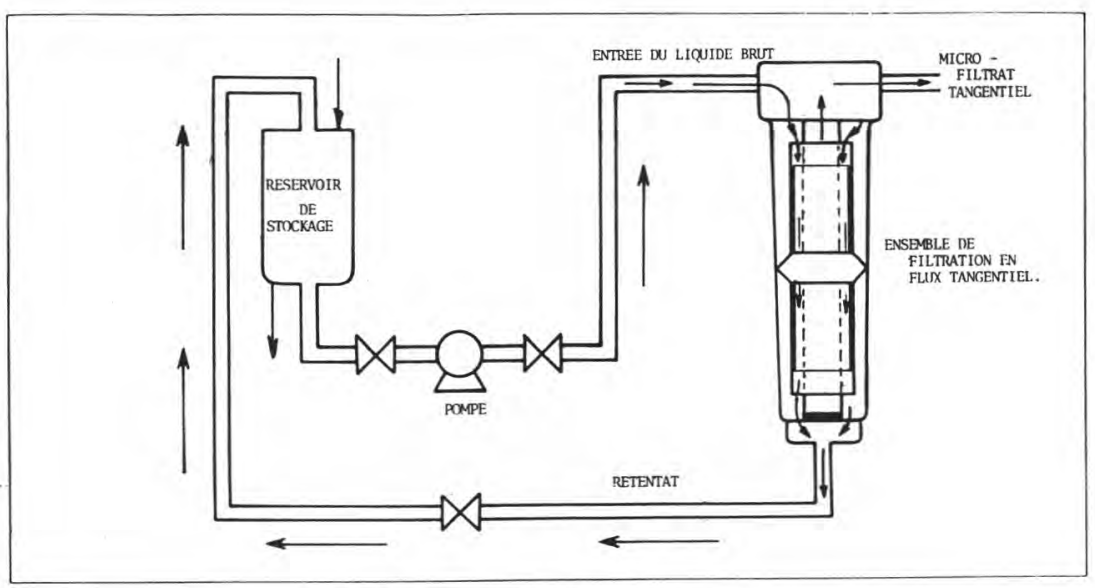

- SCHIPMA DE FONCTIONNEMENT DUI FLIX TANGENTIEL -

fig. 9

Schéma du fonctionnement du flux tangentiel.

Cross-flow filtration schema.

Il est ainsi obligé de longer les plis de la cartouche entre la gaine extérieure et l'âme centrale, et est recyclé par la pompe.

Le microfiltrat tangentiel ressort ici par la partie haute.

Des vannes à l'entrée du liquide brut, à la sortie du filtre et à la sortie du microfiltrat, permettent à la fois de régler les débits, les $\Delta \mathrm{P}$ et d'assurer très facilement le décolmatage de la couche de polarisation.

Pour de l'eau de ville par exemple, un débit de recirculation de $2 \mathrm{~m}^{3} / \mathrm{h}$ permet, avec une cartouche de $250 \mathrm{~mm}$ de hauteur, d'obtenir un microfiltrat de $1 \mathrm{~m}^{3} / \mathrm{h}$ sous une faible perte de charge.

De plus, comme les particules ricochent pour la plupart sur la surface lisse de la membrane et que le colmatage lent est essentiellement dû à la couche de polarisation, on peut " nettoyer " facilement comme indiqué plus haut.

On a dès lors un appareil à une ou plusieurs cartouches qui peut fonctionner en automatique sans problème, à bas seuil, et est très économique.

Toutefois, si le fluide à traiter est très chargé en particules, une préfiltration sera nécessaire ou on pourra procéder par cascades d'unités à flux tangentiel, de seuils décroissants.

Il faut noter qu'une microfiltration tangentielle à $0,2 \mu$ coupe en dessous de $0,1 \mu$ et à $0,1 \mu$, coupe à environ $0,05 \mu$. A un tel seuil, on recouvre l'UF à haut poids moléculaire mais avec de forts débits. 
L'intérêt du procédé est alors évident.

Il a été mis en œuvre avec succès sur des eaux ainsi que sur des liquides alimentaires tels que le vin (voir chapitre précédent).

Il est applicable aux lactosérums avant UF, à la bière, etc.

Il faut noter en outre que le procédé de filtration tangentielle peut servir aussi bien à épurer un fluide qu'à le concentrer, suivant qu'on s'intéresse au microfiltrat ou au liquide concentré dans la boucle.

On peut ainsi concentrer des solutions, des vaccins ou des sérums.

La photo de la figure 10 montre un appareil industriel à une seule cartouche, débitant plus de $1 \mathrm{~m}^{3} / \mathrm{h}$ de microfiltrat à $0,2 \mu$, sur de l'eau.

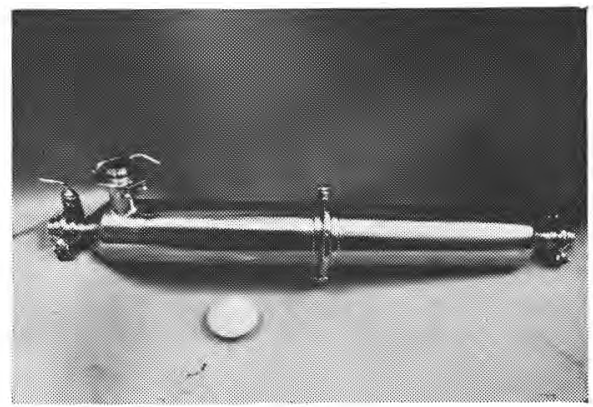

fig. 10

Photo d'un appareil à 1 cartouche filtrant en flux tangentiel : débit de plus de $1 \mathrm{~m}^{3} / \mathrm{h}$ de microfiltrat à $0,2 \mu$ sur de l'eau.

Cross-flow cartridge: water permeation flux higher than $1 \mathrm{~m}^{3} / \mathrm{h}$ with $0,2 \mu$ pore size.

\section{b) Applications en laboratoire}

Nous nous limiterons ici à des opérations de contrôle rapide, pour lesquelles les caractéristiques suivantes des membranes Nuclépore en polycarbonate ou en polyester sont mises en avant :

- pouvoir de coupure très serré ;

- surface lisse propice à l'examen microscopique ;

- bonne transparence de la membrane, qui ne se colore pas ;

- rinçage rapide et nettoyage possible à contre-courant.

- Contrôle bactérien rapide du lait par épifluorescence

Cette méthode, connue sous le nom de D.E.F.T.*, mise au point par le National Institute For Research in Dairying, à Reading (U.K.), a été développée en France par l'I.N.R.A. et plusieurs C.I.L. 
La Société Systèmes Analytiques met à la disposition des utilisateurs une technique simple d'analyse d'images « à la carte » par épifluorescence.

Ci-dessous, nous donnons les grandes lignes de cette méthode, qui a été décrite en détail dans la revue "La Technique Laitière », nº 974 de mars 1983 et la comparons à la méthode classique actuelle.

\begin{tabular}{c}
\hline Méthode D.E.F.T. \\
Durée : $25 \mathrm{~min}$ \\
1. Dans un petit tube à essai : \\
- mettre $0,5 \mathrm{ml}$ de Bactotrypsine ; \\
- ajouter $2 \mathrm{ml}$ de Triton $\times 100$ à \\
- e,5\% en volume ; ajouter $2 \mathrm{ml}$ de lait et agiter.
\end{tabular}

2. Laisser incuber le tube à essai fermé pendant $10 \mathrm{~min}$ dans un bain-marie à $50^{\circ} \mathrm{C}$.

3. Préparer l'unité de filtration comprenant un disque $\varnothing 25 \mathrm{~mm}$, seuil $0,6 \mu$ Nuclépore polycarbonate.

4. Préchauffer l'ensemble, raccordé à un vide de $100 \mathrm{~K}$ Pascals, avec $5 \mathrm{ml}$ de Triton $\times 100$, préfiltrés à $0,2 \mu(0,1 \%$ en volume) et chauffés à $50^{\circ} \mathrm{C}$.

5. Filtrer l'échantillon de lait préparé en 1 et 2 .

6. Rincer le tube à essai avec du Triton et s'en servir pour rincer le filtre.

7. Ajouter alors le colorant $(2,5 \mathrm{ml})$ d'acridine orange et rincer à l'aide de $2,5 \mathrm{ml}$ d'acide citrique puis $2,5 \mathrm{ml}$ d'iso-propanol.

8. Démonter le disque Nuclépore, le sécher à l'air et le placer dans une huile à immersion non fluorescente entre lame et lamelle.

9. Examiner à l'aide d'un microscope à épifluorescence et compter les bactéries, soit manuellement, soit automatiquement.
Méthode boîte de Pétri

\section{Durée : 3 jours}

1. Filtration sur membrane cellulosique.

2. Mise en place de la membrane sur une boîte de Pétri.

3. Développement des bactéries pendant 3 jours.

4. Dénombrement avec microscope classique des colonies bactériennes.

* Direct Epifluorescence Filter Technique. 
L'acridine orange (ou liquide fluorochrome) provoque une fluorescence verte de l'A.D.N. et une fluorescence rouge orangé de l'A.R.N.

La couleur de la fluorescence dépend donc de la structure secondaire de la molécule d'acide nucléique. Si le fluorochrome pénètre la paroi bactérienne en concentration suffisante, les cellules en croissance, qui ont un fort rapport A.R.N./A.D.N. auront une fluorescence rouge orangé, tandis que les cellules inactives qui ont un faible rapport A.R.N./A.D.N. auront une fluorescence verte.

Il est ainsi commode de distinguer en quantité et en qualité (ce qui n'est pas possible par la méthode des boîtes de Pétri) les bactéries actives.

La méthode qui se pratique à l'aide de manifolds de 5 ou 10 cellules de filtration est certes plus onéreuse que la méthode classique, mais très abordable, compte tenu de la rapidité du contrôle et de la diminution des en cours de fabrication.

Cette méthode s'applique, moyennant quelques aménagements, aussi bien au contrôle des laits crus que des produits laitiers comme le lait pasteurisé, écrémé U.H.T. ou même le beurre et la crème.

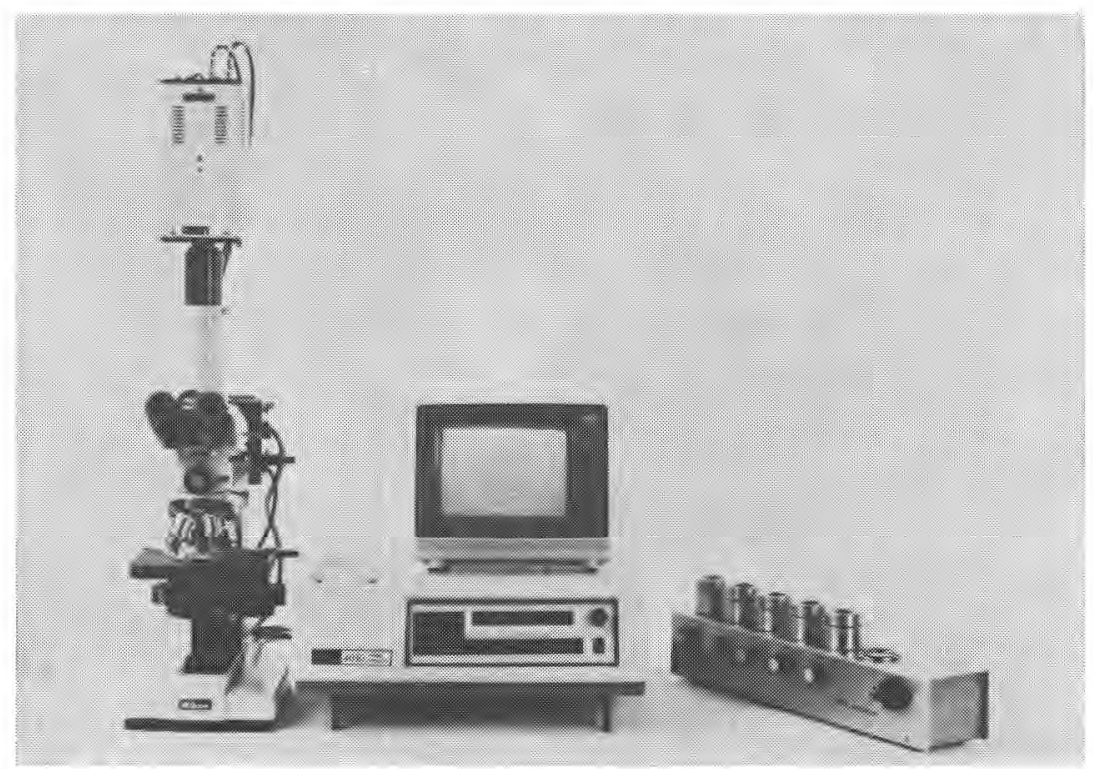

fig. 11

Microscope à épifluorescence, analyseur d'image et manifold utilisés pour la méthode D.E.F.T.

Epifluorescence microscope, picture analyzer and manifold used in D.E.F.T. method. 
Il existe un coefficient de corrélation entre la méthode D.E.F.T. et la méthode boîte de Pétri de plus de 0,9 , ce qui est satisfaisant.

Enfin, en modifiant la méthode, on peut, par filtration sur membrane de seuil $1 \mu$, procéder aussi au comptage des cellules somatiques dans le lait cru.

La figure 11 montre l'ensemble de l'appareillage nécessaire (microscope à épifluorescence, analyseur d'image, manifold) et la figure 12 montre les bactéries colorées brillantes et bien distinctes sous le microscope.

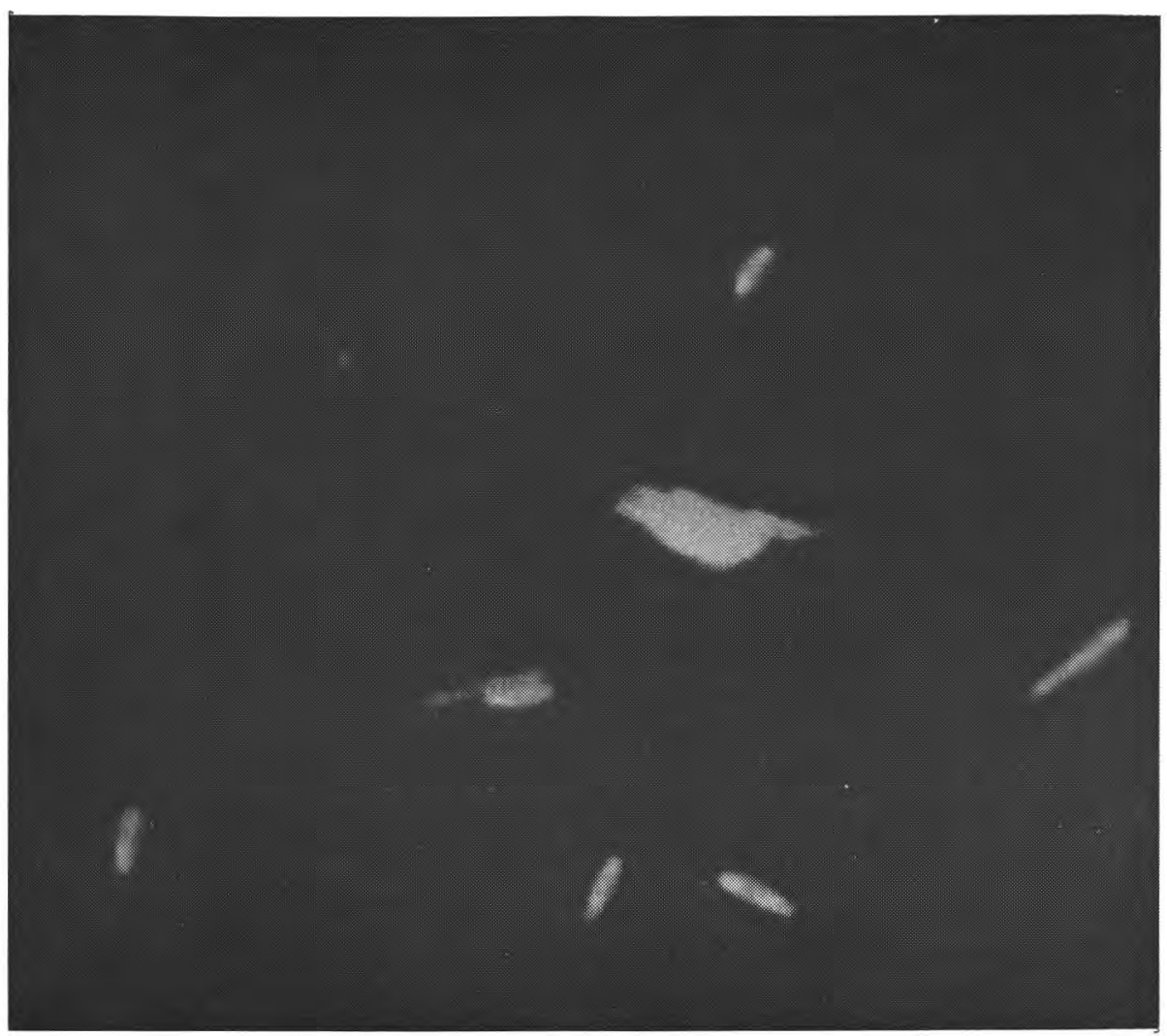

fig. 12

12 bactéries actives (rouge orangé) sur membrane Nuclépore : méthode D.E.F.T.

Red-orange coloured living bacteria on Nuclepore membrane as seen in D.E.F.T. method. 
- Contrôle bactérien rapide de l'eau

Dans ce cas, on utilise une membrane Nuclépore de seuil $0,2 \mu$ et non $0,6 \mu$.

L'échantillon n'est pas préparé, mais simplement filtré et coloré à l'acridine orange.

Le comptage s'effectue ensuite sous microscope à épifluorescence, et là aussi existe une très bonne corrélation avec la méthode boîte de Pétri.

Cette méthode est actuellement pratiquée notamment à l'Université de Bretagne Occidentale de Brest, sur de l'eau de mer.

Enfin, il faut noter, et ceci est très important, que selon les mêmes principes, on adapte cette technique au contrôle rapide du vin et de la bière.

\section{CONCLUSION}

Nous avons vu les caractéristiques qui différencient les membranes écrans des membranes filtrant en profondeur.

Grâce à la mise en valeur pratique, tant en industrie qu'en laboratoire, de ces qualités intéressantes, de nouvelles techniques ont vu le jour.

Elles apportent une grande fiabilité et une grande économie dans l'industrie grâce à l'automatisme possible (et seules ces membranes le permettent) du décolmatage à contre-courant pour des seuils aussi bas que 0,2 ou $0,1 \mu$.

Elles assurent des contrôles rapides et reproductibles au niveau bactérien, pour des coûts très convenables, contrôles rapides capables de diminuer considérablement les erreurs de fabrication.

Depuis la filtration des eaux, des vins, alcools, lactosérums, soit en direct, soit en tangentiel, aux contrôles rapides et automatiques, les membranes à pores capillaires et calibrés Nuclépore sont désormais des auxiliaires utiles des industries agro-alimentaires. 